\title{
Successful endovascular treatment of intralobar pulmonary sequestration - an effective alternative to surgery
}

\author{
Maciej Szmygin ${ }^{A, B, D, E, F}$, Krzysztof Pyra ${ }^{A, B, D, E}$, Michał Sojka ${ }^{B, D}$, Tomasz Jargiełło ${ }^{B, D}$ \\ Department of Interventional Radiology and Neuroradiology, Medical University of Lublin, Poland
}

\section{Abstract}

Introduction: Pulmonary sequestration is a rare congenital malformation characterised by the presence of non-functional and dysplastic pulmonary tissue that lacks communication with the tracheobronchial tree and has an aberrant non-pulmonary blood supply. Depending on its location, presence of the pleura covering, and venous drainage, 2 forms of pulmonary sequestration have been described: intra- and extralobar. Traditionally, surgical resection was performed; however, a growing number of cases have been treated with endovascular intervention.

Case report: A 38-year-old female patient was admitted to the hospital with severe haemoptysis for several hours. Examination at admission revealed tachycardia and tachypnoea. Computed tomography-examination disclosed the presence of an area of consolidation in the left lower lobe with a tortuous feeding artery arising from the descending aorta. Visible ground glass opacification indicated diffuse alveolar haemorrhage. Based on these findings, a diagnosis of intralobar sequestration of the left lung was made. The patient was consulted by a cardiothoracic surgeon and an interventional radiologist and qualified for endovascular treatment. In local anaesthesia femoral access was obtained and selective angiography of the common trunk of both bronchial arteries was performed. It depicted a dilated left bronchial artery supplying the sequestration and visible contrast extravasation. Embolisation of the vessel was performed with Glubran (n-butyl-cyanoacrylate). Control contrast injection showed complete elimination of the sequestration's blood supply with no residual capillary blush. Clinical improvement was observed. No complications were encountered, and the patient was discharged 7 days after the procedure.

Conclusions: Arterial embolisation is a promising alternative to surgery in the treatment of symptomatic pulmonary sequestration.

Key words: sequestration, endovascular, embolization.

\section{Introduction}

Pulmonary sequestration (PS) is a rare $(0.2-1.8 \%$ of general population) congenital malformation characterised by the presence of non-functional and dysplastic pulmonary tissue that lacks communication with the tracheobronchial tree and has an aberrant non-pulmonary blood supply. Depending on its location, the presence of the pleura covering, and venous drainage, 2 forms of PS have been described: intra- and extralobar. The former is characterised by recurrent pneumonia and is commonly seen in young adults, the latter is diagnosed in paediatric patients with lung dysfunction [1]. Traditionally, surgical resection was performed; however, a growing number of cases have been treated with endovascular intervention. We present a case of an adult patient with PS treated successfully with embolisation.

\section{Case description}

A 38-year-old female patient was admitted to the hospital with severe haemoptysis for several hours. Examination at admission revealed tachycardia and tachypnoea. Lab tests showed moderate anaemia $\left(\mathrm{RBC}-3.0 \times 10^{12}\right.$,

Correspondence address:

Maciej Szmygin, Department of Interventional Radiology and Neuroradiology, Medical University of Lublin, 8 Jaczewskiego Str., 20-954 Lublin, Poland,

e-mail:mszmygin@gmail.com 

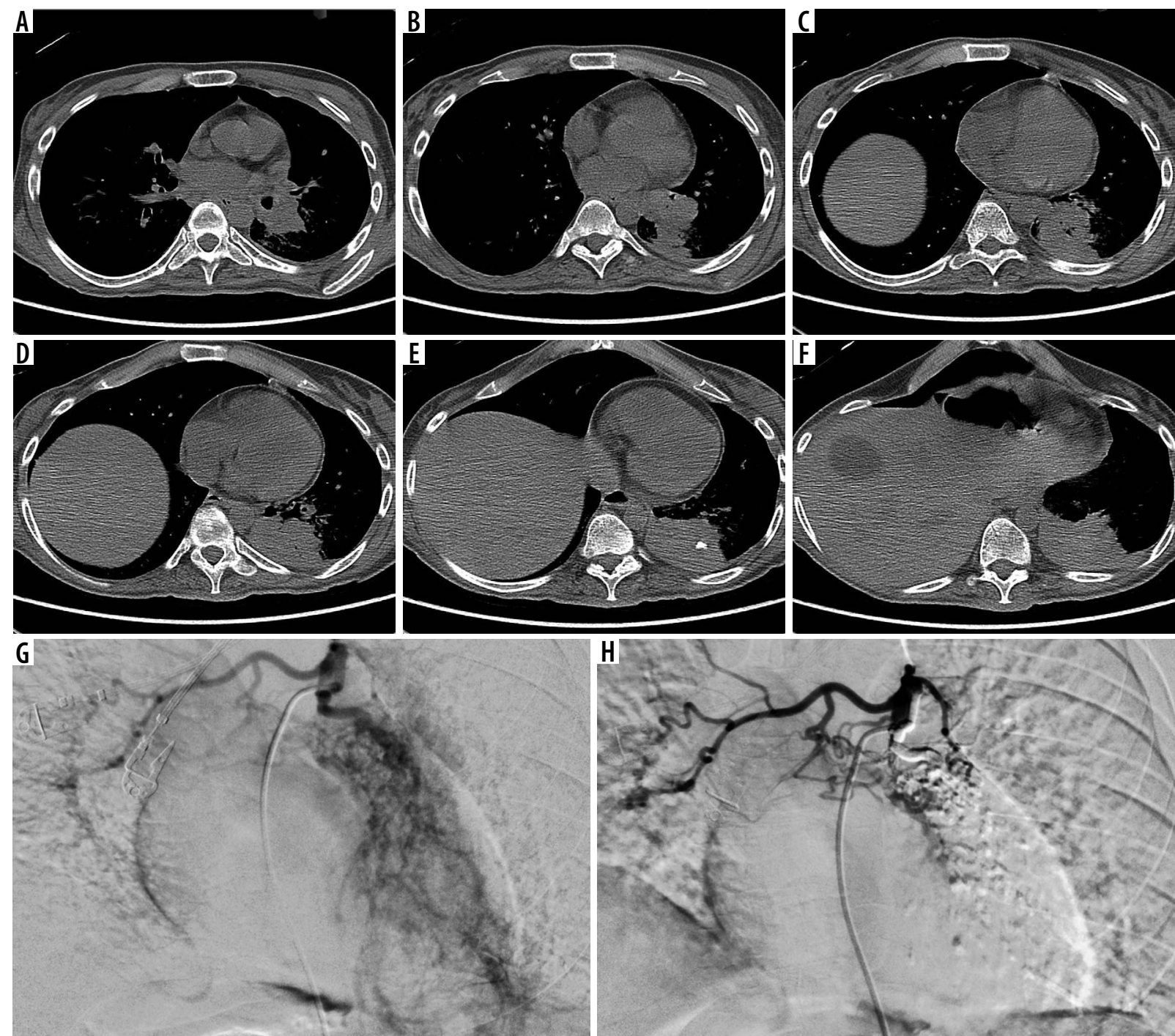

Figure 1. A-F) Axial computed tomography scans disclosed the presence of an area of consolidation in the left lower lobe. G) Initial angiography depicted a dilated left bronchial artery supplying the sequestration with visible contrast extravasation. $\mathrm{H}$ ) Control post-procedural angiography showing complete elimination of the sequestration's blood supply with no residual capillary blush. Glue cast visible

HGB - $8.7 \mathrm{~g} / \mathrm{dl}$ ). Computed tomography examination disclosed the presence of area of consolidation in the left lower lobe with tortuous feeding artery arising from the descending aorta (Figure 1A-F). Visible ground glass opacification indicated diffuse alveolar haemorrhage. Based on these finding, a diagnosis of intralobar sequestration of the left lung was made. The patient was consulted by a cardiothoracic surgeon and an interventional radiologist and qualified for endovascular treatment. In local anaesthesia femoral access was obtained and selective angiography of the common trunk of both bronchial arteries was performed. It depicted a dilated left bronchial artery supplying the sequestration and visible contrast extravasation (Figure 1G). Embolisation of the vessel was performed with Glubran (n-butyl-cyanoacrylate). Control contrast injection showed complete elimination of the sequestration's blood supply with no residual capillary blush (Figure 1H).

Clinical improvement was observed. No complications were encountered, and the patient was discharged 7 days after the procedure. Control laboratory results: $\mathrm{RBC}-$ $4.1 \times 10^{12}, \mathrm{HGB}-11.4 \mathrm{~g} / \mathrm{dl}, \mathrm{pO}_{2}-87.4 \mathrm{mmHg}, \mathrm{pCO}_{2}-$ $39 \mathrm{mmHg}$, oxygen saturation $-97 \%$.

Pulmonary sequestration is an uncommon congenital lung disease manifesting with cough, recurrent lung infections, and sputum production. Haemoptysis ranging from mild to severe results from increased blood pressure in feeding arteries leading to high capillary pressure. The current standard of care for PS is surgical resection - segmentectomy or lobectomy - performed with good outcomes and low mortality rate, especially with extralobar variant [2]. Embolisation is an endovascular technique that may be a less invasive alternative for patients with comorbidities and/or refusing surgery. Occlusion of the feeding artery causing progressive infarction and fibrosing of the sequestration seems to be equivalent to its resection. Although the majority of embolisations have been described in paediatric literature, there are only a few reports on the adult population $[3,4]$. The selection of embolisation material depends on the 
operator's preferences and experience on one side and anatomical conditions (venous drainage, presence of shunts/fistulas etc.) on the other. We chose tissue glue because it minimises the risk of future recanalisation and non-target embolisation.

\section{Conclusions}

In conclusion, arterial embolisation is a promising alternative to surgery in the treatment of symptomatic pulmonary sequestration also in adults. However, more studies are needed to firmly establish its role.

\section{Conflict of interest}

The authors report no conflict of interest.

\section{References}

1. Yucel O, Gurkok S, Gozubuyuk A, et al. Diagnosis and surgical treatment of pulmonary sequestration. Thorac Cardiovasc Surg 2008; 3: 154-157.

2. Gezer S, Taştepe İ, Sırmalı M, et al. Pulmonary sequestration: a single-institutional series composed of 27 cases. J Thorac Cardiovasc Surg 2007; 4: 955-959.
3. Ellis J, Brahmbhatt S, Desmond D, et al. Coil embolization of intralobar pulmonary sequestration - an alternative to surgery: a case report. J Med Case Rep 2018; 1: 375.

4. Zener R, Bottoni D, Zaleski A, et al. Transarterial embolization of intralobar pulmonary sequestration in a young adult with hemoptysis. J Thorac Dis 2017; 3: 188-193. 